\title{
Conocimientos sobre la competencia ortotónica desarrollados por estudiantes de Educación Básica
}

\author{
Knowledge about orthotonic competence developed by basic education \\ students
}

Recibido febrero 2021 Arbitrado marzo 2021 Aceptado abril 2021

\author{
Anadelis Córdoba \\ anadeliscr@gmail.com \\ Código ORCID: 0000-0003-0028-670X
}

Universidad Pedagógica Experimental Libertador, Caracas, Venezuela

\section{Resumen}

La investigación planteó diagnosticar cuáles fueron los conocimientos sobre competencia ortotónica que han desarrollado los estudiantes de Educación Básica. La metodología se basó en un enfoque mixto, bajo la modalidad de investigación de campo, de carácter descriptivo e interpretativo. Las técnicas usadas fueron la observación participante y la entrevista, los instrumentos el guión de entrevista y la prueba escrita. Los sujetos de estudios fueron 26 estudiantes de sexto grado de la Unidad Educativa Nacional Sorocaima. Los resultados evidenciaron que la mayoría de los estudiantes manifestaron no entender ni conocer la información concerniente a la acentuación de palabras; por lo que pudiera inferirse que no han recibido enseñanza sobre ello o en todo caso, si la hubo no se logró un aprendizaje significativo, razón por la que se diseñaron estrategias innovadoras que permitan desarrollar y afianzar la competencia ortotónica, elemento clave para el manejo adecuado de la lengua escrita.

\footnotetext{
Abstract

The purpose was to diagnose what knowledge about orthotonic competence is that Basic Education students have developed. The methodology was based on a mixed approach, under the modality of field research, of a descriptive and interpretive nature. The techniques used were participant observation and the interview, the instruments the interview script and the written test. The study subjects were 26 sixth grade students from the Sorocaima National Educational Unit. The results showed that the majority of the students stated that they did not understand or know the information concerning word stress; Therefore, it could be inferred that they have not received teaching about it or in any case, if there was, significant learning was not achieved, which is why innovative strategies must be designed to develop and strengthen orthotonic competence, a key element for management adequate written language.
}

\author{
Palabras clave: \\ Competencia \\ ortotónica; sílaba \\ tónica; lengua \\ escrita; Educación \\ Básica
}

\author{
Keywords: \\ Orthotonic \\ competence; \\ stressed syllable; \\ written language; \\ Basic Education
}




\section{INTRODUCCIÓN}

L a ortografía surge como un conjunto de normas establecidas en respuesta a los errores constantes en la escritura, durante la antigüedad. La Real Academia Española (RAE, 2010) explica que la palabra ortografía es de origen griego, y que etimológicamente dicho término hace referencia a la correcta escritura, asimismo expresa que su función esencial se centra en garantizar la comunicación escrita entre los usuarios a partir de un código común y por encima de las variantes de pronunciación que puedan derivar de factores geográficos, socioculturales, entre otros; lo cual es clave para una efectiva comunicación y entendimiento.

En ese sentido, Sánchez (2009), en uno de sus artículos, plantea que es de suma importancia dominar la ortografía como código mediador entre el emisor y el receptor para así transmitir el significado y la idea que se desea expresar de manera clara, sin ambigüedades.

Es por ello que luego del establecimiento de reglas ortográficas por la necesidad de que lo que se escribe sea entendido por el lector, la ortografía se considera de gran importancia en relación con los procesos de enseñanza y de aprendizaje, de esta manera comprender y construir diversos conocimientos y contenidos (RAE, 2010).

Una buena ortografía se hace indispensable para lograr mayor alcance y comprensión de lo leído, de tal manera que no se distorsione la idea que se desea transmitir. Por tales motivos, algunos de los principales aspectos que deben considerarse son el uso adecuado de los signos de puntuación, acorde utilización de los grafemas y muy especialmente la correcta acentuación, ya que no tildar una palabra o hacerlo de la manera incorrecta puede generar significados errados e incongruentes de lo que realmente se desea expresar.

La preocupación por el tema ortográfico es una situación que supera fronteras. La UNESCO en 2010 informó sobre un estudio realizado desde el 2008 en América Latina, en el que se determinó que de cada 10 alumnos argentinos, entre 7 y 9 presentaron escritos que dificultaron su desciframiento. Asimismo, se plantea que dichos problemas son también presentados por otros países 
(República Dominicana, El Salvador, Panamá, y Guatemala). Por último, se indicó que los enfoques que se basan en la ejecución de habilidades simples (leer, escribir, copiar, otros), no eran suficientes para la enseñanza de la composición escrita.

Lo relevante es que aún se sigue investigando sobre la competencia ortográfica. En la actualidad, existen investigaciones que evidencian que aún este tema tiene vigencia, además de que no solo están dirigidas a los primeros niveles de educación sino también al universitario y a la formación de obreros calificados, lo que refleja que hay una necesidad de trabajar por una didáctica dirigida a fortalecerla (Cofré, 2019; Marimón y Acosta, 2017; Peñalver y Borges, 2019; Rodríguez y Sánchez, 2018; Saneluterio, 2019).

En cuanto a las posibles causas de la problemática ortográfica en los diferentes países, Carratalá (s/f), catedrático de Lengua Española y Literatura en el Instituto de Educación Secundaria (IES) señala en su artículo "Las faltas de ortografía” lo siguiente:

Entre las posibles causas de las faltas de ortografía cabe mencionar la aversión por la lectura de muchos escolares, que les impide el contacto directo con las palabras. Al docente corresponde la grata tarea de ir desarrollando en los escolares una actitud favorable hacia la lectura que, sin duda, habrá de contribuir a su formación integral; lectura que, por otra parte, servirá para aumentar lo que, en términos chomskyanos, podríamos llamar la competencia lingüística de dichos escolares, pues no sólo permite la fijación visual de la ortografía de las palabras, sino también la asimilación de su significado contextual. (s.n.)

Esta situación se presenta tanto en Venezuela como en cualquier parte del mundo. En las últimas décadas, se ha observado con gran preocupación los elevados índices de la inadecuada utilización de la ortografía por la no comprensión y asimilación de ella. Situación que afecta a los estudiantes de los diferentes niveles educativos y de la cual no se escapan los profesionales de diversas áreas. Así lo refiere Morales (2001) quien plantea que los estudiantes universitarios, tanto en países desarrollados como en vías de desarrollo, no son capaces de expresarse con claridad y cohesión de manera escrita, lo cual se evidencia cuando en los textos escritos por estos se presentan grandes deficiencias 
sintácticas, semánticas, ortográficas, entre otras; imposibilitando la comprensión de los exámenes, trabajos escritos y demás materiales elaborados por ellos.

Una de las posibles causas que dan pie a las fallas ortográficas en general, sobre todo a finales del siglo pasado, es atribuible a la influencia del chat que hoy en día se encuentra en boga a partir del uso de las redes sociales como el Facebook, Twitter, Instagram, el uso de Internet y los mensajes de textos por medio del celular. Se podría decir que nos estamos enfrentando a un "nuevo lenguaje" que, lejos de enriquecer el vocabulario de los jóvenes, está ocasionando su empobrecimiento, el cual está asociado directamente con el conocimiento. En tal sentido, a la escuela se le hace cada vez más difícil la tarea para resarcir estos errores y de no corregirse a tiempo, día a día irán deformando la ortografía, y en consecuencia nuestro lenguaje, es por ello que Maurera y Jáimez (2009), en cuanto a esta dificultad exponen lo siguiente:

algunos expertos y muchos docentes cuando corrigen la ortográfica escolar confunden los códigos oral y escrito. Corrigen como disgrafía la transferencia de la conciencia fonológica al alfabeto. Este problema sería menos complejo si aún contáramos con dos únicos códigos, los tradicionales oral y escrito. Pero en el siglo XXI la situación se torna más difícil, puesto que emergen inéditos códigos, como el Chat y los mnsg de txt. En consecuencia, presumimos que a la escuela se le acumulan las tareas. (s.n.)

Sin embargo esta situación no se debe solo al chat, ni comienza en una etapa en particular sino que deriva desde los inicios cuando el niño va adquiriendo la lengua materna presentando a lo largo de su aprendizaje malformaciones y distorsiones del lenguaje que no son corregidas a tiempo. En ese sentido Balmaseda (2003) expresa

La ortografía ocupa un lugar destacado en la enseñanza de la lengua materna, porque mediante ella el alumno aprende a escribir las palabras de su vocabulario y a usar los signos de puntuación de acuerdo con las normas vigentes que le permiten decodificar y codificar textos. (p. 27)

En el aprendizaje de la ortografía, influyen además aspectos relacionados con las condiciones económicas, políticas y culturales 
dentro de la sociedad en la que el individuo interactúa y que a lo largo de la escolaridad no han sido suficientes las estrategias utilizadas por los docentes para disminuir o erradicar esta problemática, no obstante sería un error atribuir estas fallas únicamente a la escuela.

Sin embargo, en Venezuela se han tomado acciones, desde hace tiempo, para mejorar o erradicar los problemas más persistentes de escritura (uso de consonantes, signos de puntuación, uso de la tilde, entre otros), con el objeto de conseguir individuos que posean dominio idiomático, entre ellas la creación del Banco del Libro, la creación de la Comisión de Lectura, el Plan Lector, la Comisión para la Orientación de la Enseñanza y Uso de la Lengua Materna (COEULM), entre otras, aunque hoy en día algunas de estás ya no están funcionando. Adicionalmente, vale señalar, tal como lo dicen Maurera y Jáimez (2009), que muchas universidades han incorporado maestrías en Lectura y Escritura; también se han efectuado varias revisiones curriculares y diversas políticas educativas se han implantado con cada nuevo Plan de la Nación; además de un sinnúmero de congresos, jornadas y seminarios han reunidos a especialistas reimplantando ideas y acciones de índole nacional e internacional. Sin embargo, todo ello no ha sido suficiente ya que la problemática persiste.

Pese a todos los esfuerzos la situación en cuanto al problema de la escritura y por ende de la ortografía, sigue latente. Se hace necesario indagar de qué manera se puede dar respuesta oportuna y efectiva a esta situación que aqueja a las instituciones educativas del país en general y en diversos sectores.

En la Unidad Educativa Nacional Sorocaima, ubicada en el municipio Baruta, del Estado Miranda, en los últimos años ha habido un notable incremento en las debilidades ortográficas que presentan los estudiantes de Educación Básica, especialmente en cuanto a la competencia ortotónica, entendida esta como la capacidad que tiene el individuo, para acentuar correctamente las palabras que conforman su léxico, en este caso el español (Linares, 1994). Dichas debilidades, han sido preocupación tanto de los docentes como del cuerpo directivo de la institución; tomando en cuenta que son los estudiantes pertenecientes al sexto grado quienes deberían tener mayor dominio de esta competencia y quiénes están próximos a egresar para ingresar a otro subsistema, 
considerando además que el perfil del egresado contempla buen dominio de la competencia ortográfica, se decidió realizar la presente investigación.

Para indagar sobre esta problemática, se diseñó, para el año escolar 2012 - 2013, un instrumento para ser aplicado a los estudiantes y maestros de sexto grado de la institución, con el propósito de hacer un sondeo y evidenciar cuáles eran los principales problemas. Los resultados fueron los siguientes:

En primer lugar, los estudiantes manifestaron sentir tedio, dificultad y desinterés por conocer y/o comprender las reglas ortográficas y aquellos que las conocían expresaron ignorar cómo aplicarlas, tal como lo expresa Balmaseda, (2003):

los alumnos pueden manifestar: poco interés por la ortografía, insuficiente ortografía lingüística o gramatical que les impide el acceso masivo a la ortografía de las palabras... Además habría que agregar que la Ortografía como objeto de estudio- carece del atractivo que suelen tener otras materias. (p.26, 27)

En segundo lugar, enseñar las reglas ortográficas de manera descontextualizada, impide la comprensión, la adecuada transferencia y su aplicación.

En tercer lugar, los docentes generalmente consideran la enseñanza de la ortografía y su corrección, propias de la asignatura de Lengua y Literatura, sin tomar en cuenta la transversalidad a través de otras asignaturas.

En cuarto lugar, no se puede enseñar sobre lo que se desconoce, en tal sentido, algunos docentes en cuanto a las reglas ortográficas manifiestan su desconocimiento e incomprensión. En tal sentido, Villani (2005) plantea que "los docentes no tienen, en general, dominio sobre las reglas de la lengua escrita (p. 5). Esto además, se pudo evidenciar en el diseño de una guía con actividades en la asignatura de Lengua y Literatura, en la que los docentes de sexto grado cometieron errores relacionados con el uso de la tilde, clasificación de palabras según el número de sílabas, específicamente en cuanto a los términos utilizados y respecto a las secuencias vocálicas al abarcar cuatro clasificaciones en lugar de tres. 
En quinto lugar, la escritura, en función de los elementos que la norman, se ha visto influenciada por las redes sociales y el chat.

Finalmente, en sexto lugar, las insuficientes, repetitivas e inadecuadas estrategias utilizadas por el docente que es el encargado de promover el aprendizaje en sus estudiantes. Villani (2005) considera que los docentes no utilizan estrategias didácticas motivadoras, lo cual impide solventar las dificultades ortográficas de los estudiantes.

Todo esto hace reflexionar en cuanto a las estrategias empleadas año tras año, muchas de ellas ya obsoletas, pues con las nuevas tecnologías el mundo se encuentra en constante evolución y cambio, cambio que debe llegar a las aulas a través de la implementación de diferentes estrategias novedosas y acordes con los intereses de la población a la que va dirigida.

En vista de lo anteriormente expuesto se propuso diagnosticar cuáles son los conocimientos sobre competencia ortotónica que han desarrollado los estudiantes de sexto grado de la U.E.N. Sorocaima, ubicada en Baruta - estado Miranda.

\section{Ortografía}

La RAE (2010) plantea en cuanto a la palabra ortografía, que se deriva del latín ortographia, y esta del griego ортоураı $\alpha$ que significa "recta escritura", la cual surge como un conjunto de normas que determinan cuándo y cómo deben utilizarse los símbolos convencionales establecidos para representar gráficamente el lenguaje a partir del código comunicacional de la escritura.

Al dominio general de las normas que rigen la escritura de una lengua se le llama, según Linares (1994), competencia ortográfica. Esta deriva de la competencia comunicativa definida por Hymes (1972) como aquella que abarca tanto el conocimiento de la lengua como la habilidad para utilizarla y la cual deriva a su vez de la competencia lingüística, llamada así por Chomsky (1975) al referirse a las capacidades de un hablante-oyente ideal en una comunidad lingüística homogénea, en la aplicación de conocimientos sobre su lengua.

Existe estrecha relación entre este tipo de competencias (la lingüística, la comunicativa y la ortográfica), ya que estas dan cuenta de la importancia de hablar y escribir atendiendo a un 
sistema y conjunto de normas establecidas para regular la escritura y las comunicaciones.

Sin embargo, la presente investigación hizo mayor énfasis en las habilidades referidas a la ortografía, más específicamente, en cuanto a la acentuación de palabras y reglas de uso de la tilde; con base en esto, la RAE (2010) explica que la ortografía incorpora, adicionalmente, varios subsistemas que dan cuenta del valor y uso del resto de sus elementos gráficos. Para esta investigación se tomó en consideración el subsistema relacionado con la representación gráfica del acento que incluye acento prosódico, acento gráfico o tilde, la vocal tónica y átona de la palabra, entre otros.

\section{Competencia ortotónica}

El acento desde sus inicios, según la RAE (2010), ha sufrido modificaciones, ya que las lenguas como instituciones culturales y entidades históricas experimentan cambios a lo largo del tiempo; estos han sido innovaciones y reformas, las primeras incluyen aspectos de la lengua que previamente no se representaban; las segundas son cambios realizados sobre un sistema de normas ortográficas preexistente; dentro de este tipo de cambios estarían los que buscan perfeccionar las reglas de un determinado ámbito de la ortografía como ha ocurrido con las sucesivas modificaciones realizadas en el conjunto de reglas de acentuación gráfica del español, para que se cumplan de manera eficaz los fines de la ortografía.

El subsistema relacionado con los acentos también recibe el nombre de competencia ortotónica, definida por Linares (1994) como "la habilidad que demuestra el usuario de una lengua para acentuar correctamente las palabras constitutivas de su léxico. La unidad referencial es la sílaba tónica" (p.5), es decir, todas aquellas destrezas que desarrolla y lleva a cabo el estudiante para poder acentuar las palabras.

A su vez Linares (1994) expresa sobre la competencia ortotónica que “...implica el conocimiento real de la estructura silábica en cuanto a las situaciones de acentuación presente en las palabras de nuestra lengua española." (p.5)

Este tipo de competencia, según Linares (1994), exige del estudiante que: 
1. Reconozca la sílaba tónica en una palabra.

2. Clasifique las palabras según la ubicación de la sílaba tónica.

3. Reconozca los casos especiales de acentuación de palabras.

4. Aplique los aspectos formales para acentuar las palabras correctamente.

Es por ello que la competencia ortotónica requiere del conocimiento y comprensión de ciertos conceptos (ver Figura 1), que para Cova Jaime (2016), basada en lo que según Linares (1994) se debe exigir al estudiante, se concretan en cuatro pasos esenciales: separación de la palabra en sílabas, identificación de la sílaba tónica de la palabra, clasificación de la palabra según la posición de la sílaba tónica y aplicación de las reglas de la tilde según la clasificación de la palabras en aguda, grave y esdrújula o sobreesdrújula.

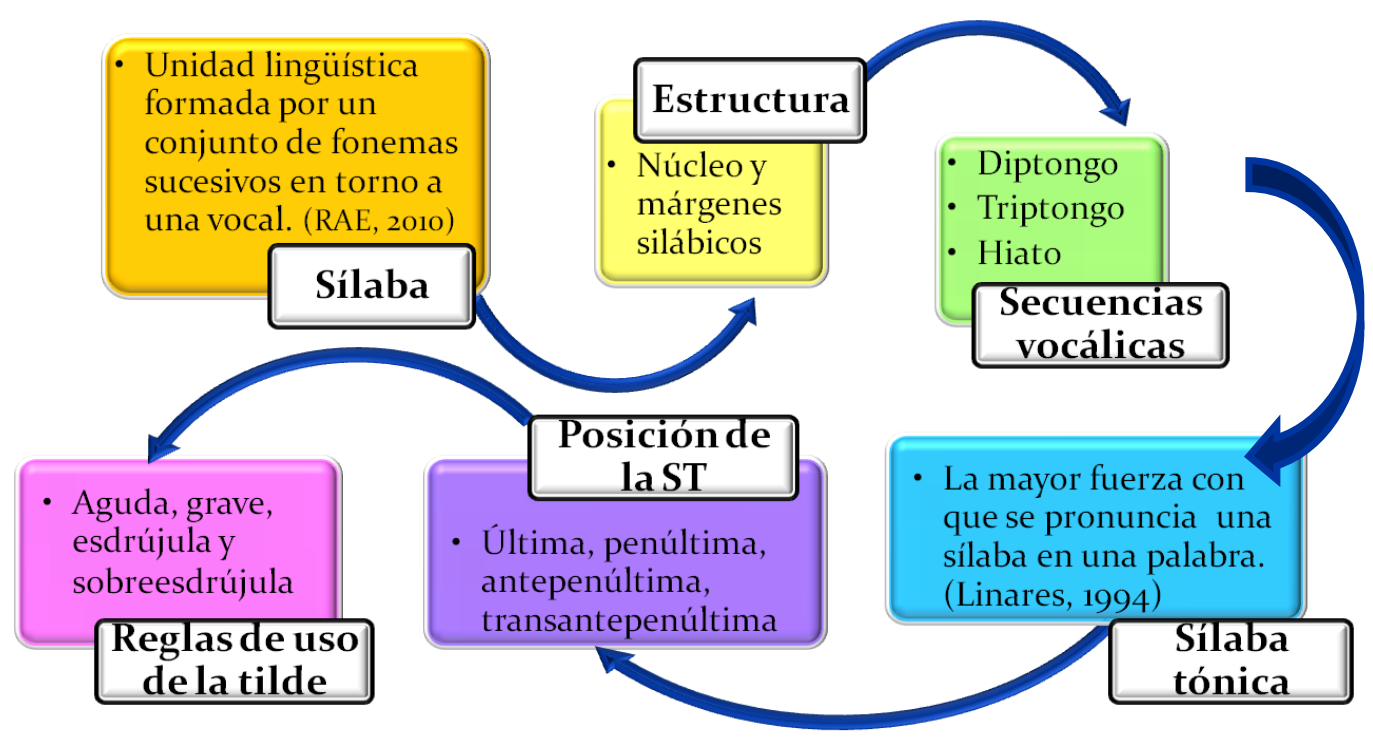

Figura 1. Conceptos. Fuente: Elaborado por la investigadora a partir de los pasos de Cova Jaime (2016).

\section{La sílaba}

En cuanto a la sílaba, Linares (1994) manifiesta que su "carácter es originalmente fonológico y segmental." (p. 2), mientras que para la RAE (2010) es la "unidad lingüística estructural de carácter fónico, formada generalmente por un conjunto de fonemas 
sucesivos que se agrupan en la pronunciación en torno a un elemento de máxima sonoridad, que en español es siempre una vocal" (p. 196).

La RAE (2010) hace una clasificación de dos tipos de sílabas tónicas, "las que portan el acento léxico o primario, y átonas, las que carecen de él” (p. 199).

\section{Estructura de la sílaba}

En la estructura de la sílaba puede distinguirse según la RAE (2010) el núcleo, que en español sólo puede estar constituido por elementos vocálicos, y dos márgenes silábicos consonánticos, en los que uno precede al núcleo y el otro lo sigue. El núcleo es el único componente imprescindible para constituir una sílaba en español y

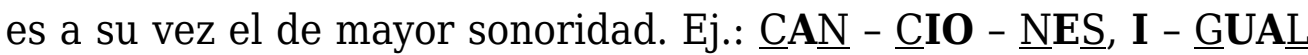
- DAD, TIEM - PO. En negrilla se identifica el núcleo y subrayado los márgenes silábicos.

\section{Secuencia vocálica y separación en sílabas}

La RAE (2010) expone que dentro de una misma palabra pueden encontrarse secuencias de hasta cinco vocales seguidas, que pueden formar o no parte de la misma sílaba. Cuando en una misma sílaba se pronuncian dos vocales seguidas forman lo que se llama "diptongo", ej. cui - da - do, puen - te; cuando se pronuncian tres vocales seguidas se forma un "triptongo", ej. Guai - re, es tu - diáis; mientras que si las vocales se pronuncian en sílabas distintas, a la secuencia vocálica se le denomina "hiato", ej. pe tró - le - o, te - a - tro.

\section{La sílaba tónica}

Definida por la RAE (2010) como "la mayor prominencia con la que se emite y percibe una sílaba con respecto a las de su entorno" (p. 190), asimismo en cuanto a la sílaba tónica expresa que es aquella que porta el acento léxico o primario, por el contrario las átonas, las que carecen de él. por su parte Linares (1994) la define como "la mayor fuerza con que se pronuncia una sílaba en una palabra” (p. 5). 


\section{El acento}

Mosterín (1981), en cuanto al acento, expone que es "un refuerzo intencional en la pronunciación de una determinada sílaba. Cuando acentuamos una sílaba, la articulamos de un modo más preciso y enérgico, y empleamos más fuerza muscular en pronunciarla..." (p. 161)

El acento se ubica siempre en la sílaba tónica y viceversa, por lo que ambos se encuentran vinculados, no obstante aunque no existe una clasificación para la sílaba tónica, si para el acento, la cual a continuación se detalla.

\section{Acento prosódico}

El acento prosódico es definido por la RAE (2010) como aquel que "pone de relieve una determinada sílaba, formada, por lo general, por más de un fonema, con respecto a las sílabas de su entorno". (p.191), por su parte Linares (1994) expone en cuanto al acento prosódico "es un acento de pronunciación, es la mayor intensidad o fuerza con que se pronuncia una sílaba en una palabra" (p. 6). Ej. aMOR, CAsa, investiGAR, renaCER, aGUAdo, entre otras.

Entonces se puede decir que significa lo mismo hablar de acento que hablar de acento prosódico.

\section{Tilde}

Según la RAE (2010), la tilde "consiste, en español, en una rayita oblicua que desciende de derecha a izquierda (') y que, colocada sobre una vocal, indica que la sílaba de la que dicha vocal forma parte es tónica". (p. 213). Ej. Teléfono, periódico, lápiz, camión.

Para ello existe un conjunto de reglas que determinan cómo y cuándo ha de utilizarse este signo.

\section{Reglas de acentuación gráfica o tilde}

Según Linares (1994) para acentuar las palabras ortográficamente se debe considerar la clasificación de palabras según la sílaba tónica: 
a) Palabras agudas: se acentúan con la tilde, si terminan en las consonantes "n", "s" o en vocal. Ej. Con tilde: camión, compás, durmió, entre otras; sin tilde: arroz, comer, televisor, entre otras.

b) Palabras graves o llanas: se acentúan con tilde cuando terminan en consonante siempre que estas no sean la " $\mathrm{n}$ " o " $\mathrm{s}$ ". Ej. Con tilde: lápiz, nácar, árbol, entre otras; sin tilde: conciencia, hijo, principiante, compraron, entre otras.

c) Palabras esdrújulas: siempre se acentúan con la tilde. Ej. Lápida, tráfico, periódico, intérprete, entre otras.

d) Palabras sobreesdrújulas: al igual que las esdrújulas siempre se acentúan con la tilde. Ej. Anótaselo, míramelo, manténgaselo, entre otras.

En tal sentido, los conceptos antes expuestos dan cuenta de todos los aspectos inherentes a la competencia ortotónica, los cuales fueron abordados a través de estrategias de enseñanza que sirvieron de herramienta a la investigadora para promover en los estudiantes la comprensión, el aprendizaje y la aplicación de las reglas ortográficas relacionadas.

\section{MÉTODO}

$\mathrm{E}$ xisten muchas maneras de abordar una investigación, una de ellas es aquella que se desarrolla bajo un diseño mixto, en la que se conciben tanto aspectos de la investigación cualitativa como de la cuantitativa, que según Hernández, Fernández y Baptista (2003) se combinan ambas metodologías a lo largo de la investigación o al menos en varias de sus etapas y a pesar de crear mayor complejidad al diseño de estudio también ofrece las ventajas de ambos enfoques.

Para Gómez y Rojas (2010), la investigación cualitativa se encarga de describir la realidad tal como la experimentan los informantes, estudia sus relaciones sociales, se basa en un modelo de interpretación sujeto - objeto, siendo sistemas de gran complejidad, en el que el segundo de ellos considera muchas partes que interactúan entre sí y con el todo, para tratar de explicar las 
razones de los diversos aspectos de comportamiento de los miembros de una comunidad.

Martínez (1998) la define como aquella que "trata de identificar la naturaleza profunda de las realidades, su estructura dinámica, aquella que da razón plena de su comportamiento y manifestaciones." (p. 8)

Por su parte, la investigación cuantitativa es la que permite responder las preguntas de la investigación, según Hernández et al. (2003), a través de "la recolección de datos para probar hipótesis, con base a la mediación numérica y el análisis estadístico, para establecer patrones de comportamiento y probar teorías" (p.5)

Asimismo, esta investigación es de carácter descriptivo e interpretativo porque a partir de la observación se describieron e interpretaron los hechos ocurridos en el escenario de la investigación.

En cuanto al diseño de la investigación Arias (1999) manifiesta "El diseño de investigación es la estrategia que adopta el investigador para responder al problema planteado" (p. 20).

El presente trabajo se sustentó bajo el diseño de la investigación de campo. La investigación de campo es descrita por el Manual de Trabajos de Grado de Especialización y Maestría de la UPEL (2016) de la siguiente manera:

Se entiende por Investigación de Campo, el análisis sistemático de problemas en la realidad, con el propósito bien sea de describirlos, interpretarlos, entender su naturaleza y factores constituyentes, explicar sus causas y efectos, o predecir su ocurrencia, haciendo uso de métodos característicos de cualquiera de los paradigmas o enfoques de investigación conocidos o en desarrollo. (p. 14)

Otra definición en cuanto a la investigación de campo es la señalada por Arias (1999) cuando expresa que "consiste en la recolección de datos directamente de la realidad donde ocurren los hechos, sin manipular o controlar variable alguna" (p. 21).

En tal sentido, la investigación de campo permitió recolectar información directamente de la realidad para conocer la problemática presente en cuanto al dominio de la competencia ortotónica de los estudiantes. 
En cuanto a las técnicas de recolección de datos, Paz (2003) afirma lo siguiente "podríamos hablar de la observación, la entrevista, etc., como prácticas de investigación o bien como estrategias de obtención de información" (p. 141).

Arias (1999) define "Las técnicas de recolección de datos son las distintas formas o maneras de obtener la información. Son ejemplos de técnicas; la observación directa, la encuesta en sus dos modalidades (entrevista o cuestionario), el análisis documental, análisis de contenido, etc." (p. 25)

A su vez Cisterna (s.f.) plantea:

Dentro del campo de la investigación cualitativa, los métodos y técnicas de investigación son bastante amplios, y el propio desarrollo de las ciencias sociales contribuye periódicamente a su enriquecimiento. Sin embargo, para efectos de sus aplicaciones concretas a investigaciones educacionales, y más aún, cuando se trata de investigaciones de tesis de grado, no todos resultan factibles, ya sea que se trate de razones de tiempo, recursos o pertinencia con el objeto de estudio.

Según Martínez (1998) para recolectar información es necesario considerar aquellos criterios que permitan realizar observaciones repetidas veces, grabar, filmar, fotografiar, hacer anotaciones, conservar los documentos, entre otros, para ello se cuenta con la observación participante en donde el investigador se encuentra en contacto directo con las personas o grupos que desea investigar.

La observación es imprescindible en la investigación cualitativa; así lo refieren Hernández et al. (2003) cuando expresan "La observación es formativa y constituye el único medio que se utiliza siempre en todo estudio cualitativo". (p. 418)

A pesar de que son muchas las técnicas de recogida de información, para efectos de esta investigación se trabajó con la técnica de la entrevista, la cual, según Rojas (1995), se utiliza cuando no se tiene suficiente información sobre lo que se desea investigar; y la observación en dos (2) modalidades, observación cualitativa entendida por Hernández et al. (2003) como aquella en la que no solo se toman notas de lo ocurrido en la práctica social, sino que conlleva a una reflexión permanente ante los hechos observados, de igual manera se trabajó con la observación 
estructurada, definida por Hernández et al. (2003) como aquella en la que los instrumentos utilizados para recabar información poseen aspectos ya establecidos sobre lo que se desea observar. Estas técnicas permitieron obtener información referida a la competencia ortotónica de los estudiantes, así como del proceso de enseñanza y de aprendizaje.

En referencia a los instrumentos, Arias (1999) plantea lo siguiente "Los instrumentos son los medios materiales que se emplean para recoger y almacenar la información. Ejemplo: fichas, formatos de cuestionario, guías de entrevista, lista de cotejo, grabadores, escalas de actitudes u opinión (tipo Likert), etc." (p. 25)

Ruiz (2002) manifiesta en cuanto a la utilización de la prueba diagnóstica y los resultados que esta arroja que esto permite al investigador la toma decisiones, relacionada con la retroalimentación de los estudiantes y profesores, así como dar cuenta de las fortalezas y debilidades presentes en la enseñanza y el aprendizaje.

Por su parte, el guión de entrevista, se compone de preguntas abiertas sobre el tema y los indicadores que se desean explorar (Rojas, 1995).

Para efectos de esta investigación se trabajó con la prueba escrita y el guión de entrevista, ya que estos instrumentos permitieron recolectar la mayor información posible y así se evitó perder información importante para el logro de los objetivos planteados.

Para poder recolectar información durante el desarrollo de una investigación se deben considerar los sujetos de estudio. Según Cisterna (s/f.), referente a los sujetos de estudio manifiesta que son aquellas personas concretas con quienes se trabaja durante el desarrollo de la investigación.

Los sujetos de esta investigación corresponden a 26 estudiantes, 15 niñas y 11 niños pertenecientes a la sección "A" del sexto grado del turno de la mañana en la U.E.N. Sorocaima ubicada en Baruta, estado Miranda, en edades comprendidas entre los 11 y 13 años.

Se logró con ello recabar información valiosa que permitió hacer un diagnóstico sobre la competencia ortotónica de los estudiantes de sexto grado de esa institución, con el fin de que esta información fuera un insumo para diseñar, en el corto plazo, 
estrategias de enseñanza innovadoras a favor del desarrollo y consolidación de dicha competencia, tan necesaria para mejorar la comprensión de la producción escrita.

Vale destacar que los instrumentos utilizados fueron evaluados a través del juicio de expertos. Para ello participaron cinco (5) expertos con grados de magíster: dos (2) de ellos en Lengua y Literatura, dos (2) en Estrategias de Aprendizaje y uno (1) en evaluación. En vista de que para Martínez (2000), en las investigaciones la validación de los instrumentos resulta necesaria.

En cuanto al análisis cualitativo, en esta investigación se realizaron los procesos de categorizar y triangular a fin de comprender e interpretar lo que estaba ocurriendo durante el proceso educativo. Para Cisterna (2005) las categorías son los tópicos por medio de los cuales se recoge y organiza la información, a su vez estas categorías se dividen en subcategorías que detallan dicho tópico en microaspectos (ver Tabla 1). En cuanto a la triangulación, Cisterna (2005) considera que debe utilizarse una vez recabada la totalidad de la información, esta consiste en reunir y cruzar toda la información pertinente con el objeto de estudio, constituyendo así la esencia del resultado de la investigación.

Tabla 1. Categorías y subcategorías.

\begin{tabular}{ll}
\hline \multicolumn{1}{c}{ Categorías } & \multicolumn{1}{c}{ Subcategorías } \\
\hline Competencia & 1. La sílaba \\
ortotónica & 2. La separación en sílaba \\
& 3. La estructura silábica \\
& 4. Las secuencias vocálicas (diptongo, hiato y triptongo) \\
& 5. La sílaba tónica (ST) \\
& 6. Las reglas de uso de la tilde según la posición de la ST.
\end{tabular}

Fuente: elaborado por la investigadora.

Con respecto al análisis cuantitativo, se realizó tomando en cuenta el porcentaje de respuestas emitidas en cada ítem o indicador de la prueba escrita. En tal sentido, se trabajó con un sistema de variables, que orientaron la temática según la visión del investigador y de estas se desprendieron a su vez ciertas dimensiones e indicadores que permitieron apreciar los aspectos más sencillos que las conformaron (ver Tabla 2). 
Tabla 2. Sistema de variables.

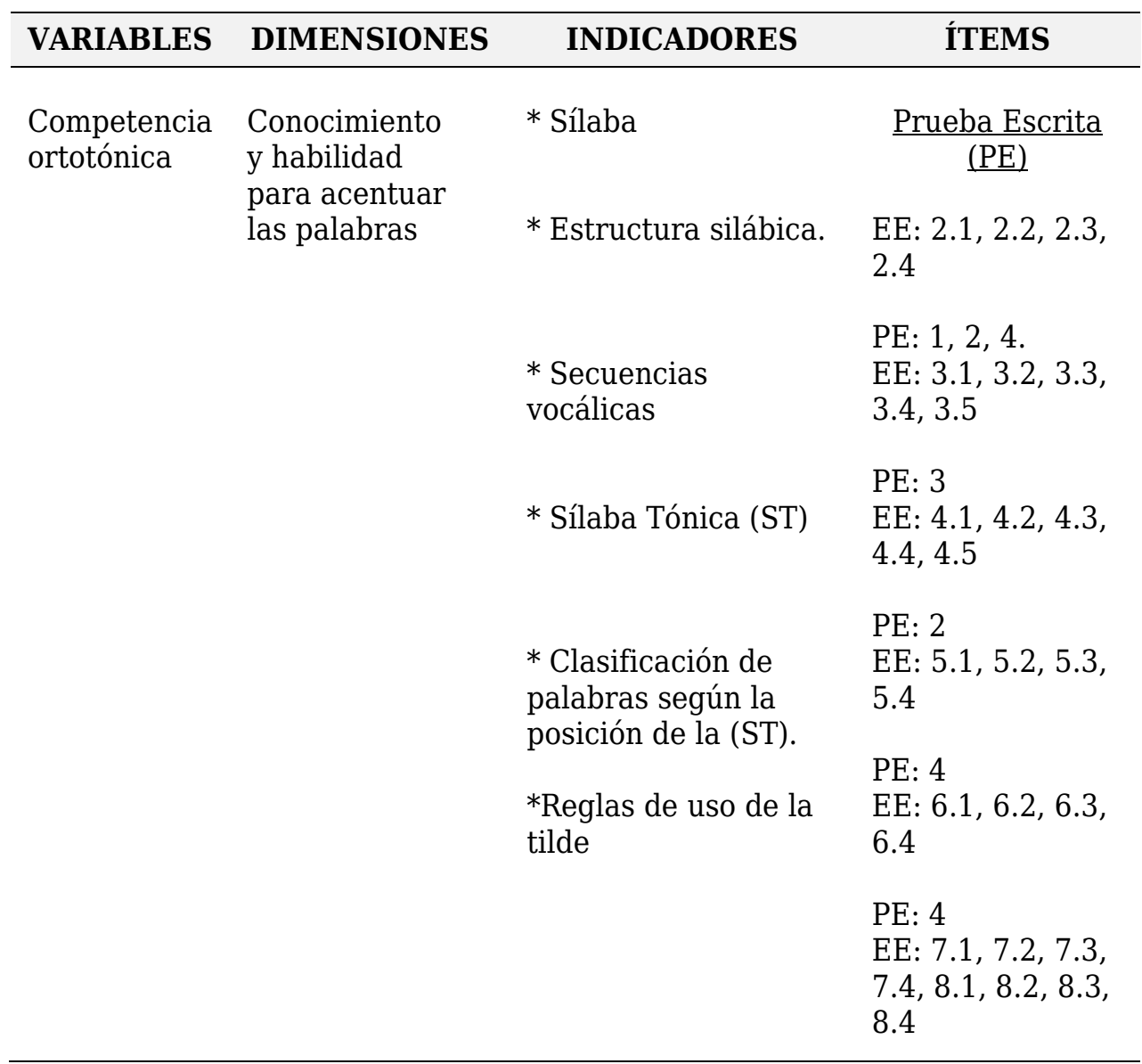

Fuente: Elaborado por la investigadora.

Con base en la información obtenida a partir del análisis cualitativo y cuantitativo se procedió a realizar la triangulación con la teoría, para poder interpretar los resultados con mayor confiabilidad.

\section{RESULTADOS}

A partir de la entrevista se recabó información sobre la categoría basada en la competencia ortotónica con la que 1 cuentan los estudiantes y esta a su vez se dividió en subcategorías, la cuales se clasificaron de la siguiente manera: 1. La sílaba, 2. La separación en sílaba, 3. La estructura silábica, 4. Las secuencias vocálicas (diptongo, hiato y triptongo), 5. La sílaba tónica (ST), 6. Las reglas de uso de la tilde según la posición de la 
ST. Esta entrevista constó de nueve preguntas que dieron respuesta a los aspectos señalados anteriormente.

Por otra parte, también se pudo indagar sobre la competencia ortotónica como variable de estudio a partir de la prueba escrita, la cual constó de la lectura de un cuento, seguida por cuatro actividades que permitieron evidenciar más adelante a través de gráficos, los resultados obtenidos, para dar respuesta a los indicadores señalados por Cova Jaime (2016): 1. Estructura silábica (ver figuras 1 y 2), 2. Secuencias vocálicas (ver Figura 3), 3. Súlaba tónica $<$ ST $>$ (ver Figura 4), 4. Clasificación de palabras según la posición de la ST (ver Figura 5), 5. Reglas de uso de la tilde (ver Figura 6).

En tal sentido, al contrastar los resultados obtenidos a través de la entrevista y la prueba escrita, se pudo conocer con mayor precisión cuál era la competencia ortotónica de los estudiantes de sexto grado.

A continuación, en la Tabla 3 se presentan los registros obtenidos a partir de la entrevista, según las subcategorías de la competencia ortotónica.

Tabla 3. Registros de la entrevista según las subcategorías de la competencia ortotónica.

\begin{tabular}{|c|c|}
\hline Subcategorías & Registros de la entrevista \\
\hline La sílaba & $\begin{array}{l}\text { Son palabras que se separan. La silaba para mi es una } \\
\text { palabra. Es para separar las palabras. La silaba se utiliza } \\
\text { para unir palabras. La silaba es la que forma a la palabra y se } \\
\text { utiliza para hacer oraciones. Su utilidad es hacer palabras. La } \\
\text { sílaba es una letra que le puedes meter a una palabra. Una } \\
\text { silaba es una palabra que uno puede agregar a una oración. } \\
\text { La silaba es una letra y una bocal que si se une con otra letra } \\
\text { y bocal forma palabras. se utiliza para hacer palabras. La } \\
\text { silaba es para completar una palabra. }\end{array}$ \\
\hline
\end{tabular}

Separación en El Proceso es saber como separar la palabra. Las dificultades sílaba es que muchas veces me equivoco. Yo lo que hago al momento de separar una sílaba es deletrearla. El proceso que devemos aser para separar en sílabas es contado. Cuando voy a separar una sílaba debo saber cuantas palabras tiene las sílabas. Yo aplaudo para ir separando las palabras. El principal proceso es contar las silabas. por ejemplo= carro ca - rro. aplaudiendo boy sacando la silaba. Las separo por paso y no tengo dificultad. 


Subcategorías $\quad$ Registros de la entrevista

Estructura silábica

Secuencias vocálicas

Sílaba Tónica (ST)

Regla de uso de la tilde según la posición de la ST
No se. No entiendo. No lo se. Es el unico idioma que se. Si porqué es una silaba cronica.

No se. No me acueldo. El triptongo es complejo. El triptongo se me ace mas facil. El triptongo se me hace mas difícil. El hiato es mas facil. Se algo de diptongo hiato y triptongo. Siempre se me olvida como se identifican. Es mas difícil el hiato. Hiato que hay veces que me confundo. Tritongo mi profe todavía no me la han dado. el dictongo por que ese no me lo se tan bien. Lo mas difícil es el hiato.

Yo logro identificar la palabra porque la silaba tiene mas sonido. No entiendo. Se pero no me acuerdo. Tengo dificultades no la entiendo. No logro identificar la silaba tonica porque no me la se. Identificar se me dificulta un poquito porque hay palabras que no conosco que no e escuchado o que no entiendo. Dificultad para buscar abeces la silaba tonica porque todas me suenan. No lo e visto. Si por su sonido y donde caiga la tilde hay es donde va el acento. Donde se siente la mayor fueza de boz. en donde cae la fuerza de voz. La silaba tonica se me dificulta mas que que separar en silabas.

No me acueldo. Se me dificulta colocar la tilde. no la desarrollo muy bien. es difícil colocar la tilde segun sea su composición. No se en donde va el atilde. No lo se. no me la enseñan. no se me precentan dificultades. No es difícil. Dificultad para colocar la tilde por la posición de la tónica. Noce cual es la tilde porque no la e podido entender.

Nota: La información representada en este recuadro presenta errores ortográficos y de redacción, ya que es una transcripción exacta de las respuestas de los estudiantes.

Se puede apreciar en cuanto a los resultados obtenidos a partir de la aplicación de la entrevista, que la mayoría de los estudiantes carecen de dominio del tema en lo concerniente a la acentuación de palabras, aunque poseen cierta noción de qué es la sílaba, cómo proceder a separar palabras en sílabas, qué es la sílaba tónica y cómo reconocerla, hay otros elementos que expresaban era complicado comprender o recordar para ellos como las secuencias vocálicas (diptongo, triptongo e hiato) y otros que desconocían en su totalidad lo relacionado con la estructura silábica. La mayoría manifestó no entender, no conocer; por lo que 
pudiera inferirse que no han recibido enseñanza sobre ello o en todo caso, si la hubo no se logró un aprendizaje significativo.

Todo lo anteriormente expuesto, se traduce en debilidades en cuanto a la competencia ortotónica, ya que los estudiantes expresaron poseer dificultades para aplicar las reglas relacionadas con el uso de la tilde, lo cual se constata a continuación a través de los resultados de la prueba escrita, por medio de la cual de manera práctica los estudiantes demostraron sus habilidades y destrezas en cuanto a la acentuación y los elementos que la conforman, obteniéndose lo siguiente:

En la Figura 2 se presenta el resultado sobre la habilidad que poseen los estudiantes para separar las palabras en sílabas y reconocer esta últimas. La mayoría $(42,31 \%)$ presenta deficiencias, mientras que un $23,08 \%$ se ubican en la opción regular y solo un 15, 38\% se encuentra bien; la opción excelente no obtuvo puntuación y un 19,23\% de los estudiantes no asistió este día. Es importante acotar que el conocimiento y la comprensión para separar las palabras en sílabas son componentes básicos para la acentuación de las palabras.

No Asistieron; $19,23 \%$ Excelente; 0\% Bien; $15,38 \%$

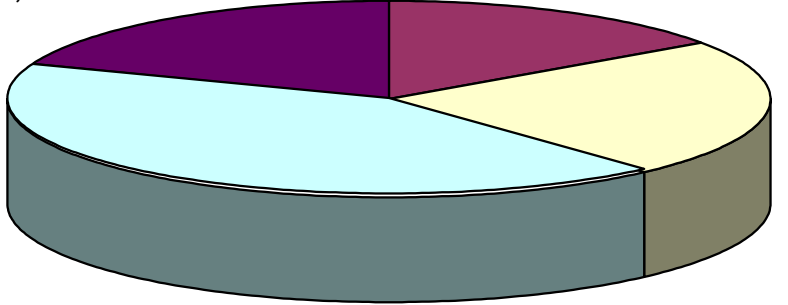

Regular; 23,08\%

Deficiente; $42,31 \%$

Figura 2. Resultados del reconocimiento de la sílaba.

En la Figura 3 un grupo minoritario de estudiantes (7,69\%) demostró encontrarse bien en cuanto al reconocimiento de la estructura silábica de cada una de las palabras, otro 15,38\% lo hizo regular; nuevamente el $19,23 \%$ no asistió a clase, mientras que un $57,69 \%$ se encontró deficiente, probando así que la mayoría del grupo tiene debilidades referente a este indicador. 


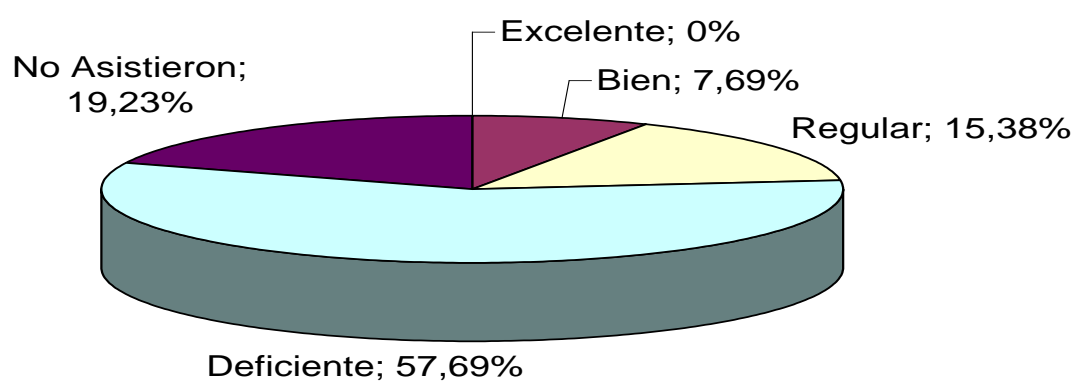

Figura 3. Reconocimiento de la estructura de cada una de las sílabas presentes en las palabras.

En la Figura 4 se observa que los estudiantes en su mayoría no identifican las secuencias vocálicas, por lo que a su vez se les dificulta establecer la diferencia entre diptongos e hiatos, ya que un $69,23 \%$ se encontró deficiente, un 11,54\% regular, el resto de los estudiantes, cifra correspondiente al 19,23\% no asistió a clase.

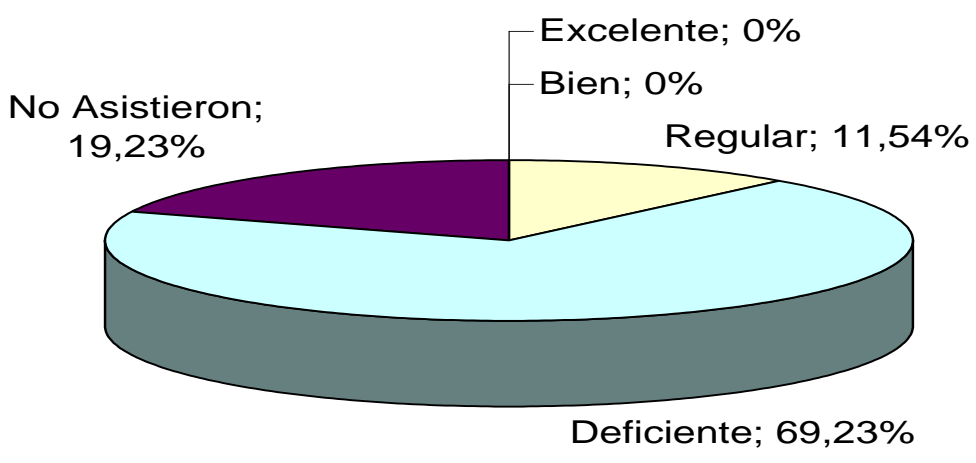

Figura 4. Identificación de las secuencias vocálicas en las palabras. Diferencias entre diptongo de hiato.

En la Figura 5 se presenta el resultado sobre la habilidad que poseen los estudiantes para reconocer la sílaba tónica en las palabras. En ese sentido ningún estudiante salió excelente, el $11,54 \%$ lo hace bien, por el contrario, la mayoría se encuentra ubicada en la opción regular con un 38,46\% y deficiente con un 30,77\%. El 19, 23\% de los estudiantes no asistió a clase ese día. Estos resultados reflejan que la mayoría de los estudiantes poseen muchas deficiencias en cuanto al reconocimiento de la sílaba tónica en las palabras. 


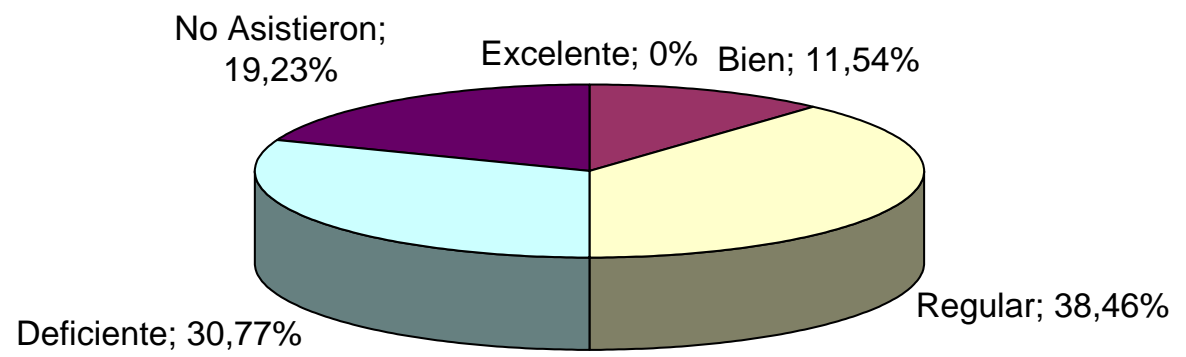

Figura 5. Identificación de la sílaba tónica presente en las palabras.

En la Figura 6 se presenta el resultado sobre la habilidad que poseen los estudiantes para clasificar las palabras en agudas, graves o esdrújulas según la posición de la sílaba tónica en las palabras. En este sentido, ningún estudiante logra clasificarlas correctamente en su totalidad. El 11,54\% lo hace bien, por el contrario la mayoría se encuentra ubicada en la opción regular con un 38,46\% y deficiente con un 30,77\%. El 19,23\% de los estudiantes no asistió ese día. Es lógico que los resultados representados en esta gráfica sean iguales que los obtenidos en la gráfica anterior, ya que si lo estudiantes no reconocen la sílaba tónica en las palabras, mucho menos lograrán ubicar en qué posición se encuentran.

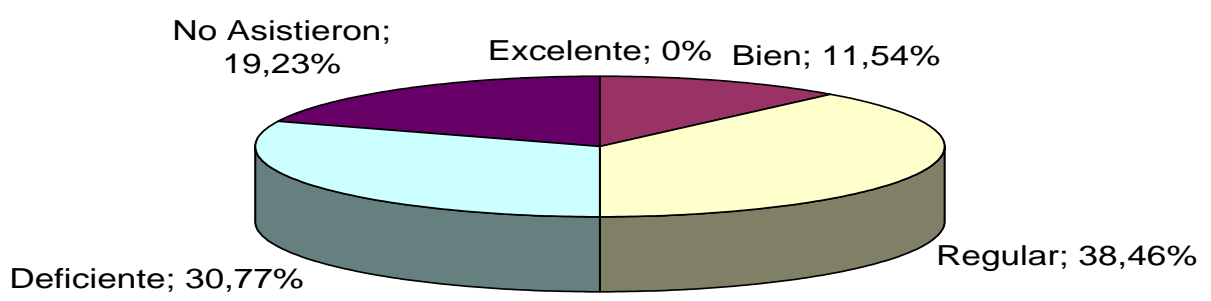

Figura 6. Clasificación de las palabras en agudas, graves, esdrújula y sobreesdrújula de acuerdo con la posición de la sílaba tónica.

En la Figura 7 se refleja que el 65,38\% de los estudiantes deja de colocar la tilde en las palabras o simplemente no la coloca en la vocal correspondiente. Una minoría del $15,38 \%$ se encuentran ubicados en la opción regular y el 19,23\% restante no asistió a clase ese día. 


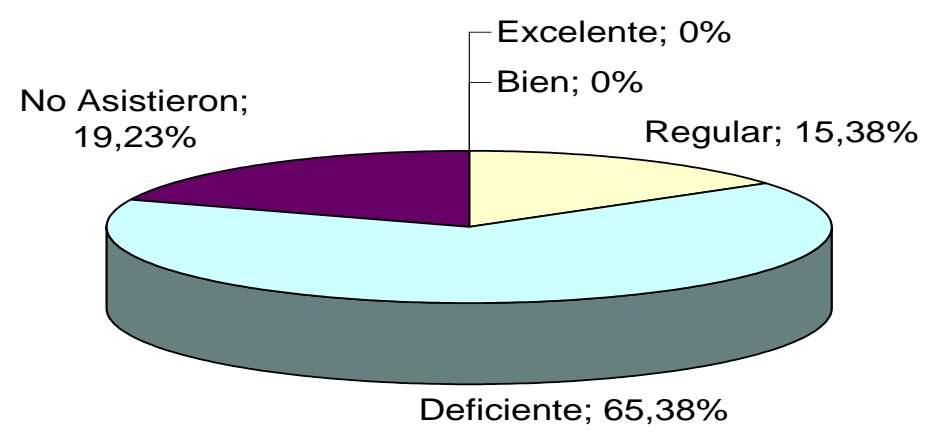

Figura 7. Aplicación de las reglas de uso de la tilde en las palabras.

En resumen se puede observar que los estudiantes desconocen la acentuación de palabras y mientras no conozcan y comprendan todos los elementos que conforman la competencia ortotónica, desde lo más sencillo (reconocimiento de la sílaba, su estructura, secuencia vocálica, sílaba tónica), difícilmente podrán comprender lo más complejo (clasificación de palabras según la posición de la sílaba tónica y la aplicación de reglas de uso de la tilde).

En tal sentido, para dar respuesta a éstas debilidades surge la II etapa de la presente investigación.

\section{CONCLUSIONES}

$\mathrm{E}$ n vista de que los estudiantes poseían vaga información en cuanto a la acentuación de palabras y los elementos que la conforman (sílaba, estructura silábica, secuencias vocálicas, sílaba tónica, clasificación de palabras según la posición de la sílaba tónica y reglas de uso de la tilde según la posición de la sílaba tónica), de hecho muchos no habían logrado desarrollar su competencia ortotónica y mucho menos habían conseguido afianzarla en el transcurso de su escolaridad, resulta necesario y urgente reflexionar en torno al desarrollo de dicha competencia, puesto que está debe ser vista como un requerimiento para la formación integral. De allí que se deban diseñar estrategias de enseñanza innovadoras que estén a favor de la promoción de una didáctica de la competencia ortotónica. 


\section{REFERENCIAS}

Arias, F. (1999) El Proyecto de Investigacion: Guía para su Elaboración. Recuperado de http://www.smo.edu.mx/colegiados /apoyos/proyecto-investigacion.pdf

Balmaseda Neyra, O. (2003). Enseñar y Aprender Ortografia. Cuba

Carratalá, F. (s.f). Faltas de Ortografía. Recuperado de http://www.aplicaciones.info/articu/arti64t.htm

Chomsky, N. (1975). Aspectos de la teoría de la sintaxis. Madrid: Aguilar.

Cisterna, F. (s.f). Métodos de Investigación en Educación. Guía Teórico Práctica. Recuperado de http://es.scribd.com/doc/57626057/55/Unidad-y-sujetos-deestudio

Cisterna, F. (2005) Categorización y Triangulación como Procesos de Validación del Conocimiento en Investigación Cualitativa. Revista Teoría. Recuperado de http://fespinoz.mayo.uson.mx/ categorizacion\%20y\%20trinagulacio\%C3\%B3n.pdf

Cofré Vera, E. D. (2019). Competencia y desempeño en ortografía de estudiantes de Educación Básica y de estudiantes en formación inicial docente (trabajo para licenciatura). Universidad de Concepción, Campus Los Ángeles, Escuela de Educación. Recuperado de http://repositorio.udec.cl/bitstream/11594/3329 /4/Cofr\%C3\%A9\%20Vera\%2C\%20Evelyn.pdf

Cova Jaime, Y. (2016). Dónde va la tilde en español. Guía en cuatro pasos. Caracas: Subdirección de Extensión, Instituto Pedagógico de Miranda José Manuel Siso Martínez. Caracas

Gómez, J. y Rojas M., de (2010) Enfoques Paradigmáticos y Métodos de la Investigación Cualitativa. Recuperado de http://www.acropolis.ujap.edu.ve/pasion-revistadigital

Hernández, R, Fernández C, Baptista, P. (2003). Metodología de la Investigación. México: Mc Graw Hill

Hymes, D. (1972). "On communicative competence. Sociolinguistics". Eds. Pride, J.B. y J. Holmes. , pp. 269-293. Londres: Penguin Books

Linares, R. (1994) El dominio ortográfico y sus competencias: Ortosilábica, Ortotónica y Ortografémica. Clave, no 3 y 4 Caracas: ASOVELE.

Marimón Ramírez, F. y Acosta Iglesias, A. (2017). La competencia ortográfica para la formación del obrero calificado en la Educación Técnica y Profesional. Revista Mendive, 15(3), 276294. Recuperado de http://mendive.upr.edu.cu/index.php/ MendiveUPR/article/view/1141/html 
Martínez, M. (1998). La Investigación Cualitativa Etnográfica en Educación. Manual Teórico Práctico. México: Trillas

Martínez, M. (2000). La Investigación Cualitativa Etnográfica en Educación. Manual Teórico Práctico. México: Trillas

Maurera S., Jaimez R., (2009). Algunas Confusiones en Lingüística Aplicada y sus Consecuencias. RLA, Revista de Lingüística Teórica y Aplicada, II Sem, 47(2)

Morales. O (2001). ¿Cómo contribuir con el Desarrollo de las Competencias de los Estudiantes Universitarios como Productores de Textos? Universidad de Los Andes. Mérida. Recuperado de http://www.postgrado.unesr.edu.ve/acontece /es/todosnumeros/num08/02_05/articulo1.pdf

Mosterín, J. (1981) La ortografía fonémica del español. Madrid: Alianza

Paz, E. (2003) Investigación Cualitativa en Educación Fundamentos Tradiciones. Recuperado de http://www.postgrado.unesr.edu.ve /acontece/es/todosnumeros/num09/02_05/capitulo_7_de_sandin.p df

Peñalver Sinclay, A. G. y Borges Damas, C. L. (2019). La competencia ortográfica para la preparación integral de las ciencias médicas. MEDISAN, 23(3). Recuperado de http://scielo.sld.cu/scielo.php?script=sci_arttext\&pid=S1029301 $92019000300557 \& \operatorname{lng}=$ es\&nrm=iso

Real Academia Española [RAE] (2010). Ortografía de la Lengua Española. Editorial Espasa Calpe, S.A.. España

Rodríguez Muñoz, F. J., y Sánchez Fernández, J. (2018). El desarrollo de la competencia ortográfica en estudiantes de Educación Secundaria. Cuadernos De Lingüística Hispánica, (31), 153-171. https://doi.org/10.19053/0121053X.n31.2018. 6095

Rojas, R., (1995). Guía para realizar investigaciones sociales. México: Plaza y Valdés

Ruiz, C. (2002). Instrumento de Investigación Educativa: Procedimiento para su diseño y validación. Barquisimeto: CIPEG

Sánchez, D. (2009). Español de la Lengua Extranjera. MarcoELE, número 09

Saneluterio, E. (2019). Didáctica de la competencia ortográfica: dos líneas de futuro. E-SEDLL $\mathrm{n} . \underline{\mathrm{o}} 2$ 2. Recuperado de https://cvc.cervantes.es/literatura/esedll/pdf/02/13.pdf

UNESCO (2010) Escritura. Un estudio de las habilidades de los estudiantes de América Latina y el Caribe. CERSE. Segundo estudio regional comparativo y explicativo: LLECE Recuperado de http://www.unesdoc.unesco.org/images/0019/001919/ 191925s.pdf 
Universidad Pedagógica Experimental Libertador. Vicerrectorado de Investigación y Postgrado. (2016). Manual de Trabajo de Grado de Especialización y Maestría y Tesis Doctorales. Caracas: Autor

Villani, M. (2005). Desarrollo de la competencia ortotónica del alumno de tercer grado de educación básica (tesis de maestría). Universidad Pedagógica Experimental Libertador. Caracas 